\title{
THE LIMIT POINTS OF A GROUP*
}

\author{
LESTER R. FORD
}

1. Introduction. The present paper is concerned with properly discontinuous groups of linear transformations. Such a group can be so transformed that the point at infinity is carried into some fixed finite region by every transformation of the group other than the identical transformation. In what follows it will be understood, without express statement, that the groups referred to have this property.

The isometric circle $\dagger$ of a linear transformation

$$
z^{\prime}=T(z)=\frac{a z+b}{c z+d}, \quad a d-b c=1, \quad c \neq 0
$$

is the circle

$$
|c z+d|=1 \text {. }
$$

The isometric circles exist for all transformations of a group other than the identical transformation. For a given group these circles all lie in a finite region and have distinct centers; and they have the property that there is only a finite number with radius exceeding a given positive quantity.

By the limit points of a group is meant the cluster points of the centers of its isometric circles. It follows from the preceding remarks that there exist isometric circles belonging to the transformations of the group which lie wholly within any circle, however small, drawn with center at a limit point.

A group is called elementary if it has no limit points, or one limit point, or two limit points. If a group has more than two limit points, its limit points form a perfect set and so are non-denumerably infinite in number. It is with these latter, or non-elementary, groups that we shall be concerned.

2. The fundamental theorem. In the study of the limit points we shall make use of the properties of the isometric circles. The various results are based on the following theorem.

* Presented to the Society, December 28, 1927; received by the editors in February, 1929.

$\dagger$ For a summary of the properties of the isometric circle see Proceedings of the National Academy of Sciences, vol. 13 (1927), pp. 286-289. For a complete treatment see the author's $A$ utomorphic Functions (McGraw-Hill Book Company, 1929), $\$ \$ 11,17$ et seq. 
TheOREM 1. Let $C$ and $K$ be circles whose centers are the limit points $P$ and $Q$ respectively of a non-elementary group. Then there exists a transformation of the group whose isometric circle lies in $C$ and the isometric circle of whose inverse lies in $K$.

Let

$$
I_{1}, I_{2}, I_{3}, \cdots
$$

be a sequence of isometric circles belonging to the group and lying in $C$ with centers

and let

$$
g_{1}, g_{2}, g_{3}, \cdots \rightarrow P
$$

$$
J_{1}, J_{2}, J_{3}, \cdots
$$

be a sequence lying in $K$ with centers

$$
h_{1}, h_{2}, h_{3}, \cdots \rightarrow Q \text {. }
$$

Let $T_{n}$ and $S_{n}$ be the transformations of the group whose isometric circles are $I_{n}$ and $J_{n}$ respectively.

Paired with each isometric circle is the circle of equal radius belonging to the inverse transformation. Thus $T_{n}$ carries $I_{n}$ into $I_{n}^{\prime}$, the isometric circle of $T_{n}^{-1}$. Let

$$
I_{1}^{\prime}, I_{2}^{\prime}, \cdots ; \quad J_{1}^{\prime}, J_{2}^{\prime}, \cdots
$$

represent the isometric circles of the inverses and let the centers of these be denoted by

$$
g_{1}^{\prime}, g_{2}^{\prime}, \cdots ; \quad \dot{h}_{1}^{\prime}, h_{2}^{\prime}, \cdots .
$$

Let $G^{\prime}$ be the set of cluster points of the set $g_{n}{ }^{\prime}$, and $H^{\prime}$ the set of cluster points of the set $h_{n}^{\prime}$. Each set has at least one cluster point, since it consists of an infinite set of points lying in a finite region.

We shall consider first the case $P \neq Q$. We may suppose (decreasing the circles, if necessary) that $C$ and $K$ are external to one another.

(1) Suppose that $G^{\prime}$ contains a point $g^{\prime}$ and $H^{\prime}$ a point $h^{\prime}$ where $g^{\prime} \neq h^{\prime}$. We can select subsequences of $g_{n}^{\prime}$ and $h_{n}^{\prime}$ approaching $g^{\prime}$ and $h^{\prime}$ respectively:

$$
g_{n_{1}}^{\prime}, g_{n_{2}}^{\prime}, \cdots \rightarrow g^{\prime} ; h_{m_{1}}^{\prime}, h_{m_{2}}^{\prime}, \cdots \rightarrow h^{\prime} \text {. }
$$

We shall make repeated use of the following lemma, which has been proved elsewhere.*

* Proceedings of the National Academy of Sciences, vol. 13 (1927), p. 290; also Automorphic Functions, \$24. 
Lemma. Let $I_{t}, I_{t}^{\prime}, I_{s}, I_{s}^{\prime}$, and $I_{u}$ be the isometric circles of $T, T^{-1}, S, S^{-1}$, and $U=T S$ respectively. If $I_{t}$ and $I_{s}^{\prime}$ are exterior to one another then $I_{u}$ is contained in $I_{\mathrm{s}}$.

Consider the transformation

$$
T=S_{m_{k}}^{-1} T_{n_{k}} ; \quad T^{-1}=T_{n_{k}}^{-1} S_{m_{k}} .
$$

For $k$ sufficiently large the isometric circles of $S_{m_{k}}^{-1}$ and $T_{n_{k}}^{-1}$, namely $J_{m_{k}}{ }^{\prime}$ and $I_{n_{k}}{ }^{\prime}$, are arbitrarily near $h^{\prime}$ and $g^{\prime}$ respectively and so are external. It follows from the lemma that the isometric circle of $T$ lies in $I_{n_{k}}$, and hence in $C$. Similarly, applying the lemma to $T^{-1}$, the isometric circle of $T^{-1}$ lies in $J_{m_{k}}$ and in $K$. This proves the theorem for this case.

(2) Points $g^{\prime}$ and $h^{\prime}$ for the preceding treatment exist unless $G^{\prime}$ and $H^{\prime}$ each consists of a single point: $G^{\prime}=H^{\prime}=\alpha$.

If $\alpha=P$ or $\alpha=Q$ the theorem obviously holds for the transformation $S_{n}$ or $T_{n}$ for $n$ sufficiently large. So we shall assume $\alpha \neq P, \alpha \neq Q$.

By taking $n$ and $m$ large enough we can make $I_{n}$ as near to $P, J_{m}$ as near to $Q$, and $I_{n}^{\prime}$ and $J_{m}^{\prime}$ as near to $\alpha$ as we wish. Hence we can choose $n$ and $m$ so that $I_{n}$ and $I_{n}^{\prime}$ are exterior to one another and $J_{m}$ and $J_{m}^{\prime}$ are exterior to one another. Now when the isometric circles of a transformation and its inverse are external circles the transformation is loxodromic or hyperbolic and each circle contains one of the fixed points of the transformation. Then $I_{n}^{\prime}$ contains a fixed point $\alpha_{n}$ of $T_{n}$ and $J_{m}^{\prime}$ contains a fixed point $\beta_{m}$ of $S_{m}$.

Suppose $\alpha_{n} \neq \beta_{m}$. Now $\alpha_{n}$ is a fixed point of $T_{n}{ }^{q}$ and $\beta_{m}$ is a fixed point of $S_{m}{ }^{q}$. If $q>1$ the isometric circle of $T_{n}{ }^{q}$ lies in $I_{n}$ and that of $S_{m^{q}}$ lies in $J_{m}$. Also the isometric circle of $T_{n}^{-q}$ contains $\alpha_{n}$ and that of $S_{m}^{-q}$ contains. $\beta_{m}$. By taking $q$ large enough we can make the radii of these circles less than $\left|\alpha_{n}-\beta_{m}\right| / 4$. Then the isometric circles of $T_{n}^{-q}$ and $S_{m}^{-q}$ are external circles.

We now apply the lemma to the transformation $T=S_{m}^{-q} T_{n}^{q}$. Its isometric circle lies in that of $T_{n}{ }^{q}$, and hence in $I_{n}$ and in $C$. Similarly, the isometric circle of the inverse, $T^{-1}=T_{n}^{-q} S_{m}^{q}$, lies in that of $S_{m}^{q}$, and so in $J_{m}$ and in $K$.

We now show that $\alpha_{n}=\beta_{m}$ is impossible for $n$ and $m$ large enough. The remaining fixed points of $T_{n}$ and $S_{m}$ are distinct since one lies near $P$ and the other near $Q$. That $\alpha_{n} \neq \beta_{m}$ then follows directly from the following proposition.

If two hyperbolic or loxodromic transformations belonging to a group have one fixed point in common but the other fixed points distinct, then the group contains infinitesimal transformations.

Let the group be so transformed that the common fixed point is at infinity. Then the transformations have the forms 


$$
\begin{aligned}
& T: \quad z^{\prime}-a=M(z-a), \quad|M| \neq 1, \\
& S: \quad z^{\prime}-b=N(z-b), \quad|N| \neq 1, \quad b \neq a ;
\end{aligned}
$$

and the powers of these transformations are

$$
\begin{aligned}
& T^{n}(z)=M^{n} z+a\left(1-M^{n}\right), \\
& S^{m}(z)=N^{m} z+b\left(1-N^{m}\right) .
\end{aligned}
$$

We now form the following product:

$$
U=T^{-n} S^{-m} T^{n} S^{m}
$$

We find, on working this out, the translation

where

$$
U=z+\omega,
$$

$$
\omega=(a-b)\left(1-M^{-n}\right)\left(1-N^{-m}\right) \text {. }
$$

By suitable choices of $m$ and $n$ we can make $M^{-n}$ and $N^{-m}$ arbitrarily small and so make the period $\omega$ arbitrarily near $a-b$.

Given $\epsilon>0$, we form two such translations

$$
U_{1}=z+a-b+\epsilon_{1}, \quad U_{2}=z+a-b+\epsilon_{2},
$$

where $\left|\epsilon_{1}\right|<\frac{1}{2} \epsilon, \quad\left|\epsilon_{2}\right|<\frac{1}{2} \epsilon$, and $\epsilon_{1} \neq \epsilon_{2}$. Then

$$
U_{1} U_{2}^{-1}=z+\epsilon_{1}-\epsilon_{2}
$$

is a translation of period $\epsilon_{1}-\epsilon_{2}$, where $0<\left|\epsilon_{1}-\epsilon_{2}\right|<\epsilon$. Since $\epsilon$ may be chosen arbitrarily small, the group contains infinitesimal transformations. It is therefore not a discontinuous group.

There remains the case $P=Q$. We wish to prove that there exists a transformation $T$ whose isometric circle and the isometric circle of whose inverse both lie in the circle $C$ surrounding $P$.

Let $C$ be made small enough that it does not contain all the limit points, and let $P_{1}$ and $P_{2}$ be distinct limit peints outside $C$. About $P_{1}$ and $P_{2}$ construct circles $C_{1}$ and $C_{2}$ exterior to one another and to $C$.

Let $T_{1}$ be a transformation such that its isometric circle $I_{1}$ lies in $C$ and that of its inverse, $I_{1}^{\prime}$, lies in $C_{1}$, and let $T_{2}$ be a transformation such that its isometric circle $I_{2}$ lies in $C$ and that of its inverse, $I_{2}^{\prime}$, lies in $C_{2}$. We now apply the lemma to the transformation

$$
T=T_{2}^{-1} T_{1}, \quad T^{-1}=T_{1}^{-1} T_{2} .
$$

Since $I_{2}^{\prime}$ and $I_{1}^{\prime}$ are exterior to one another the isometric circle of $T$ lies in $I_{1}$ and hence in $C$. Similarly, the isometric circle of $T^{-1}$ lies in $I_{2}$ and hence in $C$. This completes the proof of the theorem. 
CoROllary. Let $P$ and $Q$ be limit points of a non-elementary group. Then there exists a transformation of the group, one of whose fixed points is in an arbitrarily small neighborhood of $P$ and the other is in an arbitrarily small neighborhood of $Q$.

This follows from the fact that the fixed points of a transformation lie within or on the isometric circles of the transformation and its inverse. In this corollary $P$ and $Q$ are not necessarily distinct.

We are now able to prove the following precise theorem embodying an important property of the limit points.

Theorem 2. Let $C$ be a circle with center at a limit point $P$ of a nonelementary group, and let $Q$ be any point whatever in the plane. Then $C$ contains an infinite number of distinct points congruent to $Q$.

It suffices to prove that $C$ contains one point congruent to $Q$ and distinct from $P$; for we can then replace $C$ by a circle $C^{\prime}$ with center $P$ and small enough to exclude the first congruent point and thus get a second congruent point, and so step by step get an infinite number of distinct congruent points.

Let $P_{1}$ be a limit point lying in $C$ and distinct from $P$ and $Q$ (here $Q$ and $P$ may be identical). Such a point exists, since $P$ is a cluster point of limit points. Let $P_{2}$ be any other limit point distinct from $P$ and $Q$. Let $C_{1}$ and $C_{2}$ be circles with centers $P_{1}$ and $P_{2}$ respectively and sufficiently small that $P$ and $Q$ are exterior to both circles and that $C_{1}$ lies in $C$. Let $T$ be a transformation whose isometric circle $I$ lies in $C_{2}$ and the isometric circle $I^{\prime}$ of whose inverse lies in $C_{1}$ (Theorem 1). $T$ carries the exterior of $I$ into the interior of $I^{\prime}$. Hence $Q$ is carried into a point $Q^{\prime}$ lying in $I^{\prime}$. Then $Q^{\prime}$ lies in $C$ and is distinct from $P$; and the theorem is established.

This theorem is no longer true if we drop the requirement that the group be non-elementary. Its failure occurs in the case of an elementary group if $Q$ is a limit point.

3. Non-loxodromic groups. A properly discontinuous group is called Fuchsian if the transformations have a common fixed circle and each carries the interior of the circle into itself. It is well known that a Fuchsian group contains no loxodromic transformation. That a group without loxodromic transformations is not necessarily Fuchsian is shown by the doubly periodic group. This is, however, an elementary group. We shall prove the following

THEOREM 3. A non-elementary properly discontinuous group which contains no loxodromic transformations is a Fuchsian group.

We shall show first that all the limit points lie on a circle. Let $A, B, C$ be distinct limit points and let $K$ be the circle determined by them. We shall 
show that any other limit point $P$ lies on $K$. The necessary and sufficient condition for this is that the cross ratio

be real.

$$
\lambda=\frac{(A-B)(C-P)}{(A-C)(B-P)}
$$

The transformations

$$
\begin{aligned}
& T(z)=(a z+b) /(c z+d), \quad a d-b c=1, \\
& S(z)=(\alpha z+\beta) /(\gamma z+\delta), \alpha \delta-\beta \gamma=1,
\end{aligned}
$$

are non-loxodromic if and only if $a+d$ and $\alpha+\delta$ are real. Let $I_{t}, I_{t}^{\prime}, I_{s}, I_{\text {! }}$ be the isometric circles of $T, T^{-1}, S, S^{-1}$ respectively. The centers of these circles are $-d / c, a / c,-\delta / \gamma, \alpha / \gamma$ respectively. The first pair have radius $1 /|c|$, the second pair $1 /|\gamma|$.

According to Theorem 1 we can find transformations $T$ and $S$ belonging to the group so that $a / c,-d / c, \alpha / \gamma,-\delta / \gamma$ are arbitrarily near $A, C, B, P$ respectively and so that $1 /|c|$ and $1 /|\gamma|$ are arbitrarily small. Given $\epsilon>0$, we can choose $T$ and $S$ so that

$$
\mu=\frac{\left(\frac{a}{c}-\frac{\alpha}{\gamma}\right)\left(-\frac{\alpha}{c}+\frac{\delta}{\gamma}\right)}{\left(\frac{a}{c}+\frac{d}{c}\right)\left(\frac{\alpha}{\gamma}+\frac{\delta}{\gamma}\right)}=\lambda+\epsilon^{\prime}
$$

where $\left|\epsilon^{\prime}\right|<\frac{1}{2} \epsilon$, and also so that $\left|\epsilon^{\prime \prime}\right|<\frac{1}{2} \epsilon$, where

$$
\epsilon^{\prime \prime}=\frac{\frac{1}{c^{2}}+\frac{1}{\gamma^{2}}}{\left(\frac{a}{c}+\frac{d}{c}\right)\left(\frac{\alpha}{\gamma}+\frac{\delta}{\gamma}\right)} .
$$

Now by hypothesis $S^{-1} T$ is non-loxodromic. We find readily that the condition for this is that

$$
-\delta a+\beta c+\gamma b-\alpha d
$$

be real. Hence

$$
\nu=-\frac{-\delta a+\beta c+\gamma b-\alpha d}{(a+d)(\alpha+\delta)}
$$

is real. On dividing by $\gamma c$ and using the relations $a d-b c=1, \alpha \delta-\beta \gamma=1$, we find 
We have then

$$
\nu=\frac{\left(\frac{a}{c}-\frac{\alpha}{\gamma}\right)\left(-\frac{d}{c}+\frac{\delta}{\gamma}\right)+\frac{1}{c^{2}}+\frac{1}{\gamma^{2}}}{\left(\frac{a}{c}+\frac{d}{c}\right)\left(\frac{\alpha}{\gamma}+\frac{\delta}{\gamma}\right)}=\mu+\epsilon^{\prime \prime} .
$$

$$
\lambda=\nu-\epsilon^{\prime}-\epsilon^{\prime \prime}, \quad|\lambda-\nu|<\epsilon .
$$

Since $\nu$ is real, the imaginary part of $\lambda$ cannot exceed $\epsilon$ in absolute value, where $\epsilon$ is arbitrarily small. It follows that $\lambda$ is real, and $P$ lies on $K$.

Each transformation of the group carries the set of limit points into itself. Hence each transformation carries $K$ into itself.

It remains to show that each transformation carries the interior of $K$ into itself. Now every non-loxodromic transformation carries the interior of any of its fixed circles into itself with the exception of the elliptic transformation of period two $(a+d=0)$ with its two fixed points on the fixed circle. We shall show that no transformations of this type occur.

Let $T$ be such a transformation and let $I$ be its isometric circle. Since $T$ carries the interior of $I$ into its exterior there are limit points both within and without $I$. Let $P_{1}$ and $P_{2}$ be limit points outside $I$; and let $S$ be a transformation such that its isometric circle $I_{s}$ lies near $P_{1}$ and that of its inverse $I_{s}^{\prime}$ is near $P_{2}$, and such that $I, I_{s}$, and $I_{s}^{\prime}$ are exterior to one another.

We consider the transformation

$$
U=S T, \quad U^{-1}=T^{-1} S^{-1}=T S^{-1} .
$$

Applying the lemma we have that the isometric circles $I_{u}$ and $I_{u}^{\prime}$ lie respectively in $I$ and $I_{s}^{\prime}$ and so are exterior to one another. Both $S$ and $U$ are by hypothesis non-loxodromic and are hence hyperbolic, since the isometric circles of the transformation and its inverse are exterior to one another. Both then carry the interior of $K$ into itself. But this is impossible. Since $T$ carries the interior of $K$ into its exterior and $S$ carries the exterior of $K$ into the exterior, $S T$ carries the interior into the exterior. This contradiction rules out the existence of the elliptic transformation $T$; and the theorem is established.

4. Schottky sub-groups. A Schottky group is a group generated by linear transformations $T_{1}, \cdots, T_{n}$, where $T_{k}$ carries a circle $Q_{k}$ into a circle $Q_{k}^{\prime}$ in such a way that the exterior of $Q_{k}$ goes into the interior of $Q_{k}^{\prime}$ and where the $2 n$ circles $Q_{1}, Q_{1}^{\prime}, \cdots, Q_{n}, Q_{n}^{\prime}$ are exterior to one another. The limit points of the group lie in the $2 n$ circles and form a discrete set of points. 
TheOREM 4. Given a non-elementary properly discontinuous group $\Gamma$, and a positive constant $\epsilon$. Then there exists a Schottky sub-group $\Gamma^{\prime}$ of $\Gamma$ such that every limit point of $\Gamma$ is within a distance $\epsilon$ of a limit point of $\Gamma^{\prime}$.

It is understood here, as stated in the first paragraph of this paper, that the point at infinity is an ordinary point. Let the plane be divided into squares of side $\epsilon / 3$ by rulings parallel to the real and imaginary axes. Then only a finite number $N$ of these squares have limit points of $\Gamma$ within them or on their boundaries. Let $P_{1}, P_{1}^{\prime}, \cdots, P_{n}, P_{n}^{\prime}$ be distinct limit points of $\Gamma$ so chosen that each of the $N$ squares has one at least of the points within it or on its boundary. With $P_{1}, P_{1}^{\prime}, \cdots, P_{n}, P_{n}^{\prime}$ as centers let circles $C_{1}$, $C_{1}^{\prime}, \cdots, C_{n}, C_{n}^{\prime}$ respectively be drawn with radii sufficiently small that each circle is exterior to all the others, each radius in any case not exceeding $\epsilon / 3$.

Let $T_{k}$ be a transformation of $\Gamma$ whose isometric circle $I_{k}$ lies in $C_{k}$ and the isometric circle $I_{k}^{\prime}$ of whose inverse lies in $C_{k}^{\prime}$ (Theorem 1). Then the circles $I_{1}, I_{1}^{\prime}, \cdots, I_{n}, I_{n}^{\prime}$ are exterior to one another; and the transformations $T_{1}, \cdots, T_{n}$ generate a Schottky group.

Any limit point $P$ of $\Gamma$ is within a distance $2^{1 / 2} \epsilon / 3$ of one of the points $P_{1}, \cdots, P_{n}^{\prime}$ and each of these latter points is within a distance $\epsilon / 3$ of limit points of $\Gamma^{\prime}$. Hence $P$ is within a distance $\epsilon$ of limit points of $\Gamma^{\prime}$.

We note that the limit points of $\Gamma^{\prime}$ are also limit points of $\Gamma$.

Rice InSTITUTE, Houston, Texas 